\title{
Frontières
}

\section{La médiatisation du suicide}

Ses effets sur les comportements suicidaires

\section{Hélène Bélanger}

Volume 12, numéro 1, automne 1999

Suicides, générations et culture

URI : https://id.erudit.org/iderudit/1074517ar

DOI : https://doi.org/10.7202/1074517ar

Aller au sommaire du numéro

Éditeur(s)

Université du Québec à Montréal

ISSN

1180-3479 (imprimé)

1916-0976 (numérique)

Découvrir la revue

Citer ce document

Bélanger, H. (1999). La médiatisation du suicide : ses effets sur les comportements suicidaires. Frontières, 12(1), 100-103.

https://doi.org/10.7202/1074517ar d'utilisation que vous pouvez consulter en ligne.

https://apropos.erudit.org/fr/usagers/politique-dutilisation/ 


\section{La médiatisation du suicide Ses effets sur les comportements suicidaires}

\author{
Hélène Bélanger, \\ directrice régionale du Programme d'aide aux employés, \\ Banque de Montréal.
}

On sait que le suicide est devenu un phénomène de société. Depuis le début du siècle et plus particulièrement depuis la seconde guerre mondiale, on observe une augmentation des taux de suicide dans la plupart des pays industrialisés. Plusieurs théories fort complexes ont été élaborées pour expliquer ce phénomène. Toutefois, comme le faisait remarquer dernièrement Henry Aubin ${ }^{1}$, journaliste à The Gazette, parmi les facteurs qui contribuent à influencer les comportements suicidaires la couverture médiatique du suicide retient rarement l'attention des professionnels de la communication.

Sur le plan scientifique, les études sur la médiatisation du suicide et sur son impact sur les comportements reliés au suicide sont nombreuses et remontent à quelques décennies. Bien que les premières études se soient avérées non concluantes compte tenu des nombreux problèmes méthodologiques présents, on trouve de nos jours plusieurs recherches scientifiques indiquant que la publication ou la télédiffusion de reportages sur le suicide contribue à en accroître le nombre.

Pour mesurer l'impact de la couverture médiatique sur le suicide, il faut prendre en considération le phénomène de contagion des comportements suicidaires. Phillips ${ }^{2}$ fut le premier auteur à démontrer scientifiquement l'existence de ce phénomène et à préciser les caractéristiques inhérentes aux reportages qui peuvent contribuer à la propagation du suicide. Afin de mieux comprendre les mécanismes par lesquels les médias influencent les comportements suici- daires, il faut concevoir le suicide comme un concept appris et socialement transmis. Dans cette optique, la théorie de $\mathrm{Kral}^{3}$ sur le développement du concept du suicide apparaît des plus intéressantes.

\section{UN CONCEPT SOCIALEMENT TRANSMIS}

Kral part du principe que le suicide est une idée, néfaste en soi, mais une idée issue des mêmes croyances et des mêmes normes sociétales qui déterminent les attitudes face à l'avortement, à la sexualité, au divorce, à la politique, aux habitudes de consommation et à la mode. Le suicide, comme les notions de vie et de mort, est un concept appris.

Pour cet auteur, le suicide est une idée qui se transforme en une option consciente de s'enlever la vie lorsqu'une personne fait face à une réalité douloureuse perçue comme étant intolérable. Lidée de suicide ne jaillit pas spontanément à l'esprit; c'est au contraire un choix appris, une alternative retenue ici et maintenant comme la seule pouvant libérer la personne de sa souffrance.

Pour qu'une telle idée devienne une option acceptable aux yeux d'une personne souffrante, d'un groupe, d'une cohorte ou des membres d'une culture, il faut qu'elle soit supportée par des croyances et des normes culturelles. Selon Kral, ce n'est ni la dépression, ni l'anxiété, ni aucun autre état de détresse psychologique qui causent le suicide. Ces états peuvent en effet favoriser l'apparition d'idées suicidaires et alimenter le développement d'une crise suicidaire mais:

ce qui cause le suicide, c'est

l'adoption du suicide comme une option pour mettre fin à la douleur plutôt que de choisir une autre alternative ${ }^{4}$.
L'influence des normes et des croyances culturelles sur les comportements suicidaires est mise en évidence dans une recherche récente dirigée par Mäkinen ${ }^{5}$. Ce chercheur note que l'attitude face au suicide et à l'euthanasie a un lien direct avec les taux de suicide relevés dans les pays européens étudiés. Il constate des taux de suicide plus élevés dans les pays qui adoptent une attitude plus tolérante face aux comportements visant à encourager l'euthanasie et le suicide. Dans ces pays, les lois se rapportant à l'assistance et à l'encouragement au suicide sont effectivement moins sévères.

Comme les médias, plus particulièrement la télévision, sont maintenant devenus des outils de socialisation importants, des instruments qui reflètent les valeurs de la société à laquelle nous appartenons, il n'y a qu'un pas à faire pour comprendre le rôle qu'ils peuvent jouer sur l'acceptation et les comportements reliés au suicide.

\section{COMPORTEMENTS D'IMITATION}

Les reportages sur le suicide incitent à des comportements d'imitation. La première étude rigoureuse consacrée à l'influence des reportages journalistiques sur la propagation des comportements suicidaires est attribuée à Phillips ${ }^{6}$. L'auteur note que le taux national de suicide augmente significativement pendant une brève période suite à la publication d'un reportage sur le suicide dans les journaux. Plus le fait est publié, plus le taux de suicide s'accroît; cette augmentation s'observe de façon plus nette dans la zone géographique de distribution du journal. La démonstration scientifique de l'existence du phénomène de contagion a été faite par Phillips et il dénomma «Effet de 
Werther» l'influence de la suggestion sur les comportements suicidaires.

Cette étude a suscité un intérêt relativement à l'interprétation des caractéristiques inhérentes aux reportages et à leurs effets sur le suicide. Aussi peut-on aujourd'hui affirmer que la parution d'une nouvelle sur un suicide en première page d'un journal augmente considérablement le risque de contagion ${ }^{7}$, que la fréquence de diffusion de la nouvelle a aussi un effet incitateur ${ }^{8}$ et que le contenu du reportage peut inciter des comportements d'imitation du geste suicidaire ${ }^{9}$.

La majorité des recherches sur la couverture médiatique du suicide ont été des études épidémiologiques. Les difficultés inhérentes à la construction d'un schème expérimental qui respecte l'éthique dans ce domaine peuvent expliquer la nature de ces études. Malheureusement, les résultats tirés de ce type de recherche peuvent s'avérer difficiles à interpréter.

Toutefois, deux études rigoureuses publiées sur les effets de contagion du suicide dans le métro de Vienne mettent en relief le rôle que peuvent avoir les médias sur la prévention du suicide ${ }^{10}$. Le taux de suicide dans le métro de Vienne, inauguré en 1978, demeura très bas jusqu'en 1984. Suite à l'augmentation impressionnante des suicides au cours de l'année 1984, les journaux autrichiens se mirent à publier des reportages sensationnels sur les suicides survenus dans le métro. Alertée par l'accroissement continu de ce type de suicides, l'Association autrichienne de la prévention du suicide a rédigé en 1987 un manuel destiné aux journalistes et a demandé explicitement aux journaux de la ville de mettre en pratique les recommandations contenues dans ce recueil. Le nombre de reportages ultérieurs sur le suicide publiés par ces journaux a nettement diminué. Lorsqu'il y avait publication d'une nouvelle sur un suicide, celle-ci se limitait à un bref compterendu et était le plus souvent imprimée dans des endroits discrets, dans les pages intérieures du quotidien. On constata une baisse de $13 \%$ du taux global de suicides dans la ville de Vienne. La relation frappante entre la modification des reportages publiés sur les suicides dans le métro de Vienne et la baisse observée du nombre de suicides dans cette ville confirme l'hypothèse que les reportages sur les suicides peuvent encourager des suicides additionnels.

Par ailleurs, certains chercheurs soucieux de mieux comprendre la relation existant entre les reportages médiatiques et le suicide se sont intéressés non pas à l'influence des médias sur le taux de suicide, mais plutôt à celle des taux de suicide sur les reportages subséquents publiés dans les médias ${ }^{11}$. Ces études démontrent que le taux de suicide n'a aucun impact sur le nombre d'articles publiés sur le suicide. La décision de publier un reportage sur le suicide relève en grande partie de l'intérêt que peut susciter le récit auprès des lecteurs.

\section{VULNÉRABILITÉ ET RISQUES DE CONTAGION SUICIDAIRE}

Des recherches sur la communication de masse indiquent que l'effet des reportages sur la population varie selon des facteurs bien déterminés tels que la provenance et la nature du message, sa transmission directe ou indirecte et les différences individuelles que présentent les interlocuteurs. Ainsi, l'influence sélective des médias sur des groupes et des individus fait en sorte que quelquesuns sont plus attentifs à certains messages, s'en souviennent mieux et $\mathrm{y}$ réagissent plus facilement.

Phillips et Carstensen ${ }^{12}$ observent dans le cadre de leur étude que le taux de suicide varie chez différents groupes démographiques après la télédiffusion d'un film traitant du suicide. Aucune augmentation du taux de suicide ne fut observée dans le groupe d'individus âgés de 30 à 39 ans alors que l'impact le plus important fut noté auprès des adolescents. Dans une autre étude, les mêmes auteurs font une démonstration de la présence du phénomène de contagion suicidaire chez les adolescents ${ }^{13}$. Il est fréquemment rapporté dans la littérature scientifique que c'est parmi les adolescents que l'on observe le plus de phénomènes de contagion après un suicide. Hafner et Schmidtke ${ }^{14}$ observent que ce phénomène se manifeste particulièrement chez les jeunes de 15 à 19 ans qui présentent des problématiques similaires à celles décrites dans les reportages. Selon Kral ${ }^{15}$, il y a coïncidence entre le phénomène de contagion et la suggestibilité propre aux jeunes de cet âge, en plein processus d'identification et d'intégration sociale.

Biblarz et al. ${ }^{16}$, dans une étude faite auprès de jeunes universitaires, rapportent que les personnes qui réagissent le plus intensément à la projection d'un film sur le suicide sont celles qui s'identifient le plus au personnage qui se suicide. Non seulement les réactions émotionnelles et physiologiques sont-elles plus intenses chez ces spectateurs mais ceux-ci modifient leur perception du suicide et l'envisagent comme une alternative acceptable après avoir vu le film. Ce qui rend cette recherche d'autant plus intéressante, c'est le fait que les spectateurs invités à la présentation du film avaient été évalués au préalable relativement au risque suicidaire et à leurs opinions sur le suicide; aucun n'avait vécu de crises suicidaires ou ne considérait le suicide comme une éventualité. Ainsi, même une personne qui ne présente pas ou peu de risques suicidaires peut, après avoir été exposée à un récit sur le suicide, réagir avec une intensité émotionnelle qui la rend vulnérable au point de considérer le suicide comme une alternative acceptable.

Ces recherches indiquent que certaines couches de la population, particulièrement les adolescents, sont sensibles aux informations diffusées sur le suicide et sont vulnérables à un effet d'entraînement. Il semble que l'un des mécanismes sous-jacents au phénomène de contagion soit l'identification à des problèmes de vie similaires à ceux attribués à la personne suicidaire. Les résultats présentés dans la dernière recherche laissent toutefois entrevoir la possibilité que des personnes qui n'ont jamais été mises en contact avec le suicide peuvent être influencées par des récits sur le suicide et considérer le suicide comme une option acceptable pour faire face aux difficultés de la vie.

\section{UN EXEMPLE DE TRAITEMENT DU SUICIDE DANS LES JOURNAUX QUÉBÉCOIS}

Depuis quelques années, plusieurs personnes très connues au Québec se sont enlevées la vie. Ces suicides ont été amplement traités par les médias. Le journaliste Henry Aubin ${ }^{17}$ questionne la pertinence du nombre excessif de reportages diffusés lors du décès de célébrités québécoises ainsi que la nature des commentaires rapportés. Il relève, entre autres, un certain nombre de propos qui justifient, excusent ou endossent le geste suicidaire, et il critique également les valeurs présentes dans la société québécoise qui incitent les personnes à renoncer facilement devant les difficultés.

exprimé par le journaliste supporte les hypothèses que nous avons présentées relarivement à l'influence des valeurs culturelles sur les comportements suicidaires. Pour vérifier les observations de Henry Aubin, nous avons recensé les reportages parus dans quelques quotidiens publiés en français au Québec, à la suite du suicide de Gaétan Girouard, survenu le 14 janvier 1999.

Comme monsieur Aubin, nous avons pu constater l'abondance des reportages publiés sur le décès de Gaétan Girouard. 
La nouvelle du suicide a fait la manchette de la plupart des quotidiens. Quelques titres sensationnels et intimistes ont été relevés dans $L a$ Presse ${ }^{18}$, mais ceux-ci ne sont pas représentatifs de ce qui a été titré dans la plupart des quotidiens. Plusieurs articles spéculaient sur les motifs du suicide. Certains journalistes mettaient l'accent sur les poursuites entamées contre TVA et monsieur Girouard et la crainte que celui-ci avait de perdre son emploi ${ }^{19}$. D'autres faisaient allusion à son perfectionnisme et à un épuisement professionnel ${ }^{20}$. Enfin, quelques-uns mettaient en relief sa séparation ${ }^{21}$. Les détails entourant les heures précédant le suicide ainsi que la découverte de son corps par ses collègues de travail ont alimenté un certain nombre d'articles. Contrairement aux observations de monsieur Aubin, nous n'avons relevé que quelques articles présentant des commentaires tolérants face au geste posé par Gaétan Girouard. Ces commentaires étaient émis par des proches qui n'endossaient pas le suicide mais se limitaient à accepter le fait qu'il ait mis fin à sa $v^{2}{ }^{22}$. De nombreux articles replaçant le geste suicidaire dans son contexte et abordant la complexité de la souffrance sous-jacente à la crise suicidaire ont été publiés ${ }^{23}$.

Dans l'exemple cité, nous constatons qu'il y a eu des efforts pour présenter une couverture médiatique sobre et responsable. Plusieurs informations sur le processus suicidaire, la vulnérabilité des personnes en crise et les ressources vers lesquelles elles peuvent s'adresser ont été transmises. Ces efforts contribuent non seulement à bien informer le public mais aussi, espérons-le, à minimiser les effets de contagion du suicide sur la population. Cependant, nous notons aussi la présence de reportages spéculatifs qui mettent l'accent sur une dimension de la vie personnelle du suicidé et qui, par le fait même, réduisent à une explication simple les raisons qui ont pu pousser la personne à s'enlever la vie. Non seulement ces informations présentent une perception erronée du suicide mais, comme nous l'avons vu, servent à alimenter la détresse des personnes à risque et peuvent produire un effet d'entraînement.

\section{TRAITER DU SUICIDE, MAIS COMMENT?}

Le suicide est sans l'ombre d'un doute un sujet qui doit être traité dans les médias. La question posée n'est pas de savoir si l'on doit parler du suicide, mais plutôt de quelle façon le traitement médiatique doit être fait pour diminuer les risques de contagion suicidaire. 
À partir de l'évidence scientifique démontrant le rôle influent des médias sur les idées et les comportements reliés au suicide, nombreux sont ceux qui recommandent que les journalistes adoptent un code de conduite régissant la diffusion des informations sur le suici$\mathrm{de}^{24}$. Cependant, la responsabilité de diffuser sur le suicide une information responsable et précise ne repose pas uniquement sur les épaules des journalistes. Les experts en suicidologie et les professionnels en santé mentale peuvent assister les journalistes dans leurs efforts pour diffuser une information adéquate. Ces experts doivent sensibiliser les professionnels de la communication en expliquant davantage le potentiel de contagion suicidaire et en soulignant les aspects de la couverture médiatique qui susceptibles de favoriser les comportements suicidaires.

Une couverture médiatique concise et factuelle demeure la meilleure façon d'aborder la problématique du suicide. Un traitement sobre du suicide, qui tient compte de la complexité du processus suicidaire et qui informe le public sur les ressources d'aide accessibles, aiderait sont des mesures qui aident à prévenir le phénomène de contagion. Par ailleurs, la présentation d'explications simplistes du suicide, la diffusion de détails sur la vie privée du ou des suicidaires, l'attribution d'un facteur unique comme cause $d u$ suicide, la diffusion excessive de l'événement suicidaire et la présentation d'une couverture sensationnelle d'un suicide sont autant de pratiques journalistiques qui contribuent à augmenter la probabilité de comportements suicidaires.

\section{Notes}

1 H. AUBIN, "Legitimizing Suicide», The Gazette, 20 janvier 1999.

2 D.P. PHILLIPS, «The Influence of Suggestion on Suicide: Substantive and Theoretical Implications of the Werther Effect», American Sociological Review, vol. 39, 1974, p. 340-354.

3 M.J. KRAL, "Suicide as Social Logic», Suicide and Life-Threatening Behavior, vol. 24 , no 3, 1994, p. 245-254.

4 Ibid.

5 I. MÄKINEN, «Are there Social Correlates to Suicide», Social Science and Medecine, vol. 44, no 12, 1997, p. 1919-1929.

6 D.P. PHILLIPS, "The Influence of Suggestion on Suicide: Substantive and Theoretical Implications of the Werther Effect», 1974, ibid.

7 D.P. PHILLIPS, «Suicide, Motor Vehicle Fatalities, and the Mass Media: Evidence toward a Theory of Suggestion", American Journal of Sociology, vol. 84, no 5, 1979, p. 1150-1174.
J. JACOBY, «Consumer Psychology: an Octennium», dans Annual Review of Psychology, M.R. ROSENZWEIG et L.W. PORTER (dir. publ.), Californie, Palo Alto, 1976, p. 331-358.

S. STACK, "The Effect of the Media on Suicide: Evidence from Japan 19551985», Suicide and Life-Threatening Behavior, vol. 26, no 2, 1996, p. 132-142.

8 S. STACK, ibid.

D.P. PHILLIPS, «Suicide, Motor Vehicle Fatalities, and the Mass Media: Evidence toward a Theory of Suggestion", American Journal of Sociology, 1979, ibid. J.H. GUNDLACH et S. STACK, "The Impact of Hyper Media Coverage on Suicide: New-York City 1910-1920», Social Science Quartely, vol. 71, no 3, 1990, p. 619-627.

D.P. PHILLIPS, «The Influence of Suggestion on Suicide: Substantive and Theoretical Implications of the Werther Effect», American Sociological Review, 1974, ibid.

M.J. KRAL, "Suicide as Social Logic», Suicide and Life-Threatening Behavior, 1994, ibid.

9 S. STACK, ibid.

M. WASSERMAN, "Imitation and Suicide: A Reexamination of the Werther Effect», American Sociological Review, vol. 49, 1984, p. 427-436.

10 E. ETZERSDORFER et al., "Newspaper Reports and Suicide», The New England Journal of Medecine, vol. 327, no 7, 1992, p. 502 .

G. SONNECK et al., "Imitative Suicide on the Viennese Subway », Social Science and Medecine, vol. 38, no 3, 1994, p. 453457.

11 K. ISHII, «Measuring Mutual Causation: Effects of Suicide News on Suicides in Japan», Social Science Research, vol. 20, 1991, p. 188-195.

I. WASSERMAN et al. "Suicide and the Media: The New York Times Presentation of Front Page Suicide Stories between 1910 and 1920», Journal of Communication, vol. 44, no 2, 1994, p. 64-83.

12 D. P. PHILLIPS et L.L.CARSTENSEN, «The Effect of Suicide Stories on Various Demographic Groups: 1968-1985», Suicide and Life-Threatening Behavior, vol. 18, no 1, 1988, p 100-115.

13 D.P. PHILLIPS et L.L. CARSTENSEN, «Clustering of Teenage Suicides after Television News Stories about Suicide», The New England Journal of Medecine, vol. 315, no 11,1986 , p. 685-689.

$14 \mathrm{H}$. HAFNER et A. SCHMIDTKE, «Do Televised Fictional Suicide Models Produce Suicides?» dans Suicide among Youth: Perspectives on Risk and Prevention, R. PFEFFER (dir. publ.), Washington D.C., American Psychiatric Press, 1989, p. 117-141.

15 M.J. KRAL, "Suicide as Social Logic», Suicide and Life-Threatening Behavior, 1994, ibid.

16 A. BIBLARZ et al., «Media Influence on Attitudes toward Suicide», Suicide and Life-Threatening Behavior, vol. 21, no 4, 1991, p. 374-384.

17 H. AUBIN, «Legitimizing Suicide», The Gazette, 20 janvier 1999.
18 C. DESJARDINS, «Mardi soir, je sentais une certaine faiblesse » raconte le père de Gaétan Girouard, La Presse, 16 janvier 1999, p. A-3; "Gaétan Girouard avait peur de perdre son travail à J.E.», La Presse, 18 janvier 1999, p. A-1.

19 Ibid.

K. LAVOIE, «Pas de pressions indues», Le Soleil, 17 janvier 1999, p. A-3.

20 C. DESJARDINS, «Des funérailles émouvantes», La Presse, 19 janvier 1999, p. A-3. PRESSE CANADIENNE GRANBY, «Vibrant hommage à Girouard», Le Droit, 18 janvier 1999, p. 15.

21 J.-P. CHARBONNEAU et M.-C. MALBOEUF, «L'animateur de J.E. trouvé mort», La Presse, 15 janvier 1999, p. A-1. I. MATHIEU, "Gaétan Girouard n'est plus», Le Droit, 15 janvier 1999, p. 27. I. MATHIEU, «Une fin tragique», $L e$ Soleil, 15 janvier 1999, p. A-1.

D. FESSOU, «Un gentleman», Le Soleil, 15 janvier 1999, p. C-3.

22 L. COUSINEAU, "Requiem pour un grand journaliste de télévision», La Presse, 15 janvier 1999, p. A-4

K. LAVOIE, "Ses collègues atterrés», $L e$ Soleil, 15 janvier 1999, p. C-3.

L. COUSINEAU, "Jocelyne sans Gaétan à compter d'aujourd'hui», La Presse, 19 janvier 1999

23 P. CANTIN, «La détresse», La Presse, 18 janvier 1999, p. A-5.

N. PETROWSKI, "L'habit de Gaétan», La Presse, 19 janvier 1999, p. C-13.

P. BRUNEAU, «Salut Gaétan», La Presse, 22 janvier 1999, p. B-3.

M.-C. MALBOEUF et M. PELCHAT, «Les hommes se suicident dans la force de l'âge», La Presse, 15 janvier 1999, P. A-4. F. PILON, «L'écoute et l'attention peuvent prévenir les suicides», Le Droit, 16 janvier 1999, p. 11; "Ceux à qui le suicide fait le plus mal», Le Droit, 16 janvier 1999, p. 11

C. DESJARDINS, "Les téléphones ne dérougissent pas à Suicide Action», La Presse, 17 janvier 1999, p. A-7.

M.-C. MALBOEUF, «Au sommet, mais bien seul», La Presse, 16 janvier 1999, p. A-3.

24 J. ASHTON, «Preventing Epidemic Suicide in Young People», The Lancet, vol. 344, no 8925, 1994, p. 768 .

C. H. CANTOR et al., "The Media and Suicide», The Medical Journal of Australia, vol. 155, 1991, p. 130-131. D. R. CHAMBERS, "Publicity and SelfDestruction", Medical Science and Law, vol. 34, no 2, 1994, p. 93-94.

H. MERSKEY, «Influence of the Media: A Powerful What», The Lancet, vol. 347, no 8999, 1996, p. 416. 